\title{
Gastric cancer below the age of 55: implications for screening patients with uncomplicated dyspepsia
}

\author{
J Christie, N A Shepherd, B W Codling, R M Valori
}

\begin{abstract}
Aims-To test the hypothesis that gastric cancer presenting with uncomplicated dyspepsia is rare below the age of 55 .

Patients and methods-The area studied was the postcode defined catchment area of a district general hospital (Gloucestershire Royal) serving a population of 280500 . An open access endoscopy service has been available in this district for more than 17 years. All cases of gastric cancer during a seven year period (1986-92) were drawn from the local pathology database. The database of the neighbouring hospital and the South West Cancer Registry were searched for missed cases from the postcoded area. Hospital and general practitioner records were retrospectively reviewed with respect to duration of symptoms, and previous consultation and investigation for dyspepsia; and alarming symptoms and signs suggestive of underlying malignancy (unexplained recent weight loss, dysphagia, haematemesis or melaena, anaemia, previous gastric surgery, palpable mass, and perforation).

Results-Twenty five of 319 cases of gastric cancer detected during the seven year period were aged less than 55 . Twenty four of these 25 patients presented with one or more suspicious symptoms or signs. Only one patient $(4 \%)$ aged less than 55 presented with uncomplicated dyspepsia. In two patients there was a delay in diagnosis of more than six months after first presenting to the general practitioner. Both these patients had significant symptoms at presentation.

Conclusion-Gastric cancer is rare below the age of $55(7.8 \%$ of all cases) and, even in the presence of established open access endoscopy, presents with suspicious symptoms or signs in $96 \%$ of cases. The age limit for screening uncomplicated dyspepsia can be raised safely to 55 . (Gut 1997; 41: 513-517)
\end{abstract}

Keywords: gastric cancer; open access endoscopy; disease prevention; disease control; general practice; epidemiology; diagnosis
Gastric cancer is the fourth most common cancer, in terms of mortality, causing nearly 10000 deaths in the UK each year. ${ }^{1}$ The success of gastric cancer screening in Japan ${ }^{2}$ has encouraged screening of selected populations in this country. Hallisey et al screened all new dyspeptics aged greater than 45 presenting to selected general practices and found early (potentially curable) lesions more frequently than historical controls. ${ }^{3}$ More recently a favourable change in the proportion of patients with early lesions was reported from Leeds. ${ }^{4}$ The early diagnosis was largely attributed to the more frequent use of endoscopy, particularly as a result of open access endoscopy. It was recommended that all patients aged greater than 40 with recent onset dyspepsia should undergo endoscopy. ${ }^{4}$ However, about $25-30 \%$ of the population suffers from dyspepsia ${ }^{5}$ and $2-4 \%$ of all new general practitioner (GP) consultations are for dyspepsia. ${ }^{6}$ Many endoscopy examinations would be required to screen all subjects with dyspepsia above the age of 45 , incurring substantial cost to save a life.

There have been no randomised trials showing that screening selected populations (such as dyspeptics) for gastric cancer saves lives. However, there is a widely held belief that screening is worthwhile: virtually all dyspepsia guidelines recommend that new or unresponsive dyspeptic patients aged greater than 45 should have an endoscopy to exclude cancer. In the age of rationing of healthcare resource ${ }^{7}$ it is unwise to rely on popularly held beliefs about the value of healthcare programmes. The purpose of this study was to determine whether beliefs about the value of screening those aged between 45 and 55 with dyspepsia are well founded.

\section{Methods}

SETTING

Gloucestershire Royal Hospital (GRH) is a district general hospital serving a well defined catchment area of West Gloucestershire. For the purposes of this study the catchment area was defined according to the postcoded areas GL1-6 and GL10-19 and included inner city and rural areas. The most reliable population estimate derives from the 10 yearly UK population census, last performed in 1991. In the
Accepted for publication 4 June 1997 


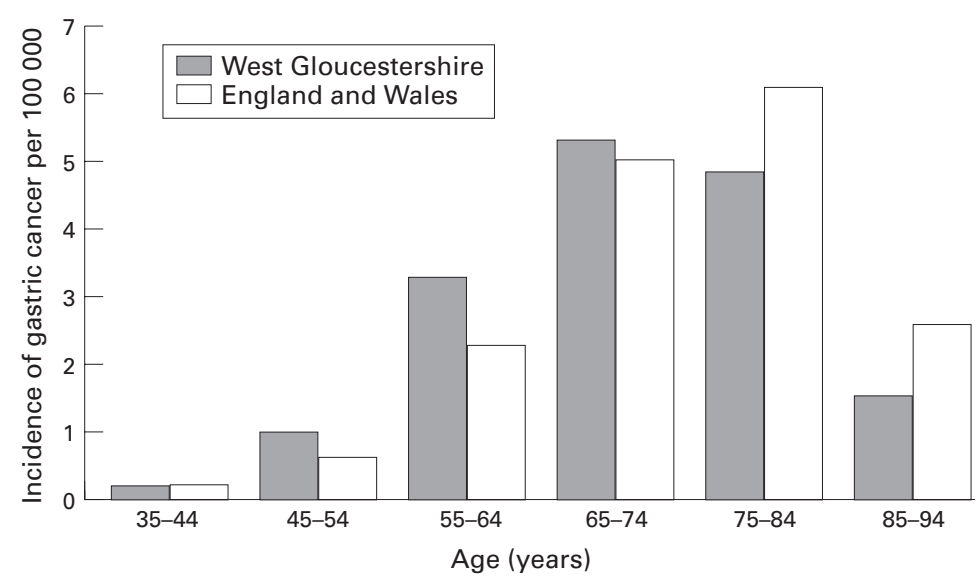

Figure 1: Incidence of gastric cancer in West Gloucestershire and in England and Wales.

census the population is defined by wards. The Gloucester, Cotswolds, and Forest of Dean Wards match the GL1-6 and GL10-19 postcoded areas almost exactly. Thus we were able to derive accurate population figures for our cohort of patients from the 1991 census figures (280 500).

Gloucestershire Royal Hospital has a well established gastroenterology service that pioneered the provision of open access endoscopy. ${ }^{89}$ It has been the local ethic to encourage early referral of dyspeptic patients for endoscopy. A fast, high quality service has meant that few patients are referred elsewhere for upper gastrointestinal investigation.

\section{PATIENTS}

Cases of gastric cancer presenting for the first time during the seven year period 1986-92 were identified from the GRH pathology database. The database contained the postcode of all patients as well as other demographic data. The diagnosis of gastric cancer had been made on clinical and pathological grounds. Cases were divided into age groups defined by the age at diagnosis. Patients outside the West Gloucestershire catchment area were excluded. The Cheltenham General Hospital pathology database (largest neighbouring district general hospital) and the South West Regional Cancer

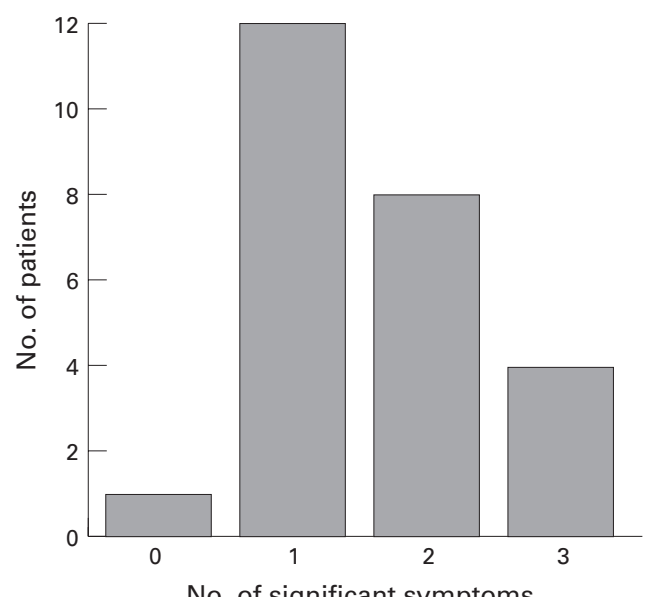

Figure 2: Frequency of suspicious symptoms or signs in patients aged less than 55 with gastric cancer.
TABLE 1 Suspicious symptoms or signs in 25 patients aged under 55 presenting with gastric cancer

\begin{tabular}{lc}
\hline Symptom & Number of patients \\
\hline Weight loss & 14 \\
Dysphagia & 8 \\
Anaemia & 7 \\
Gastrointestinal bleed & 3 \\
Previous gastric surgery & 3 \\
Palpable mass & 3 \\
Gastrointestinal perforation & 1 \\
Cerebral metastases & 1 \\
\hline
\end{tabular}

Registry were searched for missed cases by diagnosis and postcode.

To determine to what extent our incidence figures are representative of the rest of the country we compared our incidence data to those of the 1991 Office of Population Censuses and Surveys (OPCS) census for gastric cancer. ${ }^{1}$ Our data express incidence in terms of diagnosis while the OPCS data are defined in terms of death. However, as the overall five year survival of gastric cancer is only $5 \%{ }^{10}$ we believe a direct comparison is valid.

CLINICAL DETAILS

Clinical details of all patients aged less than 55 at the time of diagnosis were obtained from the hospital records and the GP records. Ten GP records had been destroyed because the patients had been dead for more than three years. Information was obtained from the GP's continuation notes and referral letter, the consultant's reply, and the house officer's preoperative clerking. Particular note was made of the time between the first consultation with the GP for dyspepsia and the diagnosis, and of symptoms or signs suggestive of malignancy.

\section{PATHOLOGICAL ASSESSMENT}

In 17 patients resections had been performed and all tissue sections were available. In another five patients sections from biopsy samples were available for typing of the tumour and assessment of Helicobacter pylori status. All tissues were routinely fixed in buffered formalin, processed through paraffin wax, and sectioned at $5 \mu \mathrm{m}$. Sections were stained with haematoxylin and eosin and $p$-aminosalicylic acid. Where non-tumorous tissue was available, sections were stained with a half Gram stain for $H$ pylori. Gastric cancers were graded according to standard criteria ${ }^{11}$ and typed according to the Lauren classification. ${ }^{12} \mathrm{Tu}-$ mours were staged according to the TNM classification. ${ }^{13}$

\section{Results}

INCIDENCE OF GASTRIC CANCER

There was a total of 319 cases of gastric cancer in West Gloucestershire during the seven year period 1986-92, giving an incidence of 16.24 cases/100 000/year. Twenty five patients $(7.8 \%)$ were aged less than 55 at the time of diagnosis. In this age group no "missed cases" were found in the Cheltenham or South West registry databases. The frequency of gastric cancer each year remained stable (41-52 cases per year). 

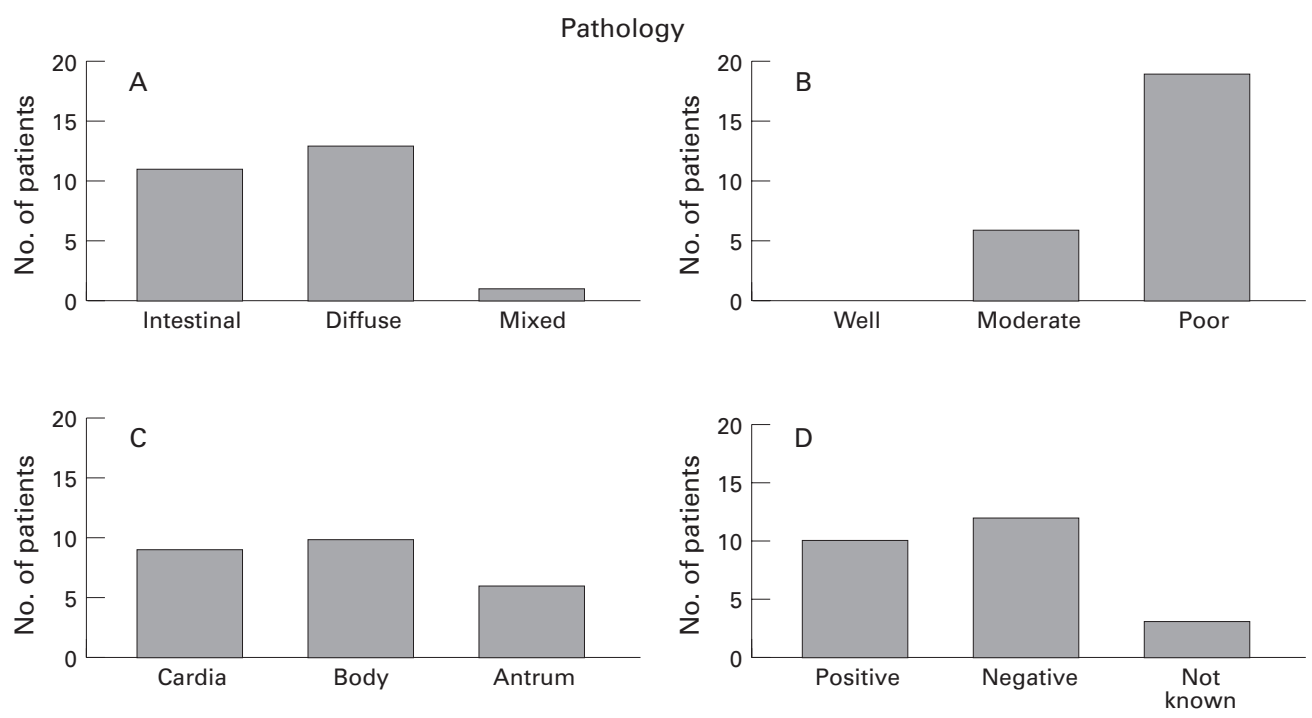

Figure 3: Pathological findings in patients aged over 55 with gastric cancer. (A) Type; (B) differentiation; (C) position; (D) H pylori status.

The incidence of gastric cancer in West Gloucestershire is comparable to that in the rest of England and Wales (fig 1). Our age bands were constructed according to age at diagnosis rather than death (OPCS). This may explain the shift of frequency to the left and the slightly higher frequency of cases aged less than 55 in our sample.

\section{COMPLICATED DYSPEPSIA}

Twenty four of 25 patients under the age of 55 had one or more suspicious symptoms or signs (complicated dyspepsia) at the time of diagnosis (table 1, fig 2). More than half the patients reported unintentional weight loss of more than $3 \mathrm{~kg}$. Six patients had weight loss of $9 \mathrm{~kg}$ or more. Of the six patients with 6-9 kg weight loss, three had other alarming features: dysphagia, anaemia (two patients), and palpable mass. Of the two with 3-6 kg weight loss one had a haemoglobin of $9.9 \mathrm{~g}$. The other had lost more than $3 \mathrm{~kg}$ unintentionally in the three weeks before presentation associated with a short history of dyspepsia. A third of patients reported dysphagia. Three patients presented with complications of the cancer without dyspepsia. Two patients had had previous vagotomy and pyloroplasty, an operation not normally considered premalignant.

\section{TIME BETWEEN FIRST GENERAL PRACTITIONER}

CONSULTATION AND DIAGNOSIS

Fifteen GP records were available for review. Ten of the 15 patients were diagnosed within three months of the first consultation for dyspepsia with their GP. In only two patients was there a delay in diagnosis beyond six months. Both patients had been referred within three months of presentation to the GP. Delay in the first patient was caused by a delay in the diagnosis of linitis plastica, despite two early endoscopies and multiple gastric biopsies. In the second patient, delay was caused by an early barium meal reported as normal. Both patients were referred early and had significant symptoms within three months of presentation to the GP.

\section{PATHOLOGY}

Histological assessment confirmed the diagnosis of gastric cancer in all 25 patients. Thirteen cases were poorly differentiated diffuse type carcinomas while 11 were of intestinal type (five moderately differentiated; six poorly differentiated). One was of mixed type (fig 3A). The majority of tumours were advanced at the time of operation (of the 17 cases where stage could be assessed one was of T1 N0 while 11 were at least $\mathrm{T} 2 \mathrm{~N} 2$ ). $H$ pylori assessment was possible in $22 / 25$ cases. Ten of these 22 cases showed evidence of $H$ pylori associated chronic active gastritis (fig 3D). In five of the negative cases only body type gastric mucosa was available for assessment. There was no evidence of atrophic gastritis or previous $\mathrm{H}$ pylori infection in the mucosa of those patients who were $H$ pylori negative at the time of cancer diagnosis. Some specimens had relatively little attached mucosa, therefore conclusions about $H$ pylori positivity in this group of patients must be drawn with care.

By 1 August 1994 only five patients were alive. One of these has metastases. The other four had resections, in 1990, 1992 (two patients), and January 1993 (diagnosis made in 1992), respectively. One of the patients operated on in 1992 was the patient presenting with uncomplicated dyspepsia. Her tumour extended through the muscularis (T2 N0) and she is currently alive and well.

\section{Discussion}

This study has shown that the prevalence of gastric cancer presenting with uncomplicated dyspepsia in the under 55 age group is very low. If our results are generally applicable there are only 25 such cases in the UK each year. The generalisability of our data depends on the following: the completeness of the data set; the time between presentation and diagnosis; the 
degree to which West Gloucestershire represents the rest of the country; and the utility of our definition of uncomplicated dyspepsia.

We believe the probability of missed cases is very low. The Gloucestershire Royal Hospital provides a service for a well defined and stable population. There has been little need for local GPs to refer their patients outside the district. Furthermore, we checked the pathology database of the neighbouring hospital (Cheltenham General) and searched for cases in the cancer registry by age and postcode (none was found).

Our inspection of the 15 GP records available indicated that there was very little delay between presentation and diagnosis. Given the information we have available on the other patients, and the ease of obtaining an endoscopy in Gloucester during the study period, we think it very unlikely that any of the other 10 cases had a delayed diagnosis. Thus in this study delay in diagnosis was not the reason why patients presented with alarming symptoms. Although the data set for GP records is incomplete we know of no comparable study that has looked at the GP records.

The social mix of West Gloucestershire is comparable to that in the rest of the country. To determine whether gastric cancer in West Gloucestershire is representative, we compared our incidence rates to those of the OPCS census for 1991 (the penultimate year of our study period). ${ }^{1}$ As the cure rate for all comers is so poor $^{10}$ the OPCS figures probably underestimate the true incidence of the disease by about $5 \%$. The similarity between our figures and those from OPCS indicates that West Gloucestershire is representative of the country.

For our definition of complicated dyspepsia to be useful the items we chose must be easily identified and not contentious. Unintentional weight loss was the most common complicating symptom occurring in more than half the patients. It is possible that the reported weight loss was exaggerated because the person reporting it (patient or doctor) knew the diagnosis. While this may be the case for the relatively marginal changes in weight ( $3 \mathrm{~kg}$ ) it is unlikely to be so for reported weight changes greater than $6 \mathrm{~kg}$. Only $2 / 14$ patients presenting with weight loss fell into the $3-6 \mathrm{~kg}$ category and one of these had anaemia. Dysphagia was the other subjective measure in our list. We think it very unlikely that dysphagia was falsely recorded because the diagnosis of gastric cancer was known. Three of our patients had gastric surgery, including vagotomy and pyloroplasty. Vagotomy and pyloroplasty is not normally considered premalignant. However, this is a relatively new operation and, in the light of our findings, we believe it must now be considered possibly premalignant. Whether it is premalignant or not, it is an easily determined, and relatively infrequent, objective measure that should prompt screening in the presence of dyspepsia.

We examined the $H$ pylori status of our patients because of the association of gastric cancer and $H$ pylori infection. ${ }^{14}{ }^{15}$ The rate of infection (10/22 evaluable samples) is relatively low. This may reflect the lack of sensitivity of our method (other methods were not used), particularly as some of the specimens had very little normal mucosa available for inspection. Alternatively, it is possible that during the premalignant phase the environment becomes hostile to the bacterium as acid levels drop. ${ }^{16}$ Our observation must be viewed with caution. However, if it is correct then absence of $H$ pylori infection cannot be used as a reason not to perform endoscopy on a patient if cancer is suspected.

Our findings are supported by those of a study from Leicester where all patients aged under 45 with gastric cancer presented with worrying symptoms or signs. ${ }^{17}$ Our study has extended this observation up to the age of 55 . Furthermore, unlike the Leicester study, we restricted our observations to a well defined postcoded area and established that there was no delay in diagnosis. Despite the limitations of the Leicester study the similarity in findings suggests that our observations are unlikely to be due to chance. A further report in abstract form from West Scotland, finding a low frequency of uncomplicated dyspepsia in the under 55 age group presenting with gastric cancer, ${ }^{18}$ supports our findings.

The belief that screening for gastric cancer of dyspeptics should begin at the age of 45 is based on the reports from Birmingham and Leeds. ${ }^{34}$ In the Birmingham study only one patient aged less than 55 had gastric cancer (presence of alarming symptoms unknown). This paper is often quoted as the definitive work justifying screening of dyspeptics over the age of 45; however, the authors state that the age limit for screening could probably be raised to 55 without adversely affecting the effectiveness of the programme. ${ }^{3}$

The Leeds group reported a "multiple time series" study ${ }^{19}$ of the staging and outcome of gastric cancer presenting to a university department of surgery. A favourable change in staging (early gastric cancer in more than $10 \%$ ) and prognosis was linked to a concurrent fourfold increase in endoscopy. It was concluded that the increase in endoscopy was largely responsible for the favourable changes. During the same period, however, there was a $50 \%$ increase in the number of patients referred for surgery. No indication is given in the paper where these extra patients were derived from. It is possible that as its reputation for treating gastric cancer developed the surgical unit in Leeds increasingly attracted referral of patients with a favourable prognosis. As well as sampling bias there are two other important biases that are impossible to eliminate without a randomised study: length and lead time bias. Length bias refers to the tendency of screening programmes to detect slow growing tumours because they are present for longer. Lead time bias refers to the tendency of screening programmes to detect tumours early without necessarily affecting their natural history. Early detection does not necessaarily mean improved survival. The Leeds study is unable to provide a representative view of the impact of early endoscopy on the staging of gastric cancer. 
We have examined the presentation and diagnosis of gastric cancer in a different way to the Birmingham and Leeds groups. ${ }^{3}$ We have established that in a well defined, stable population, gastric cancer presents late in the under $55 \mathrm{~s}$ and with significant symptoms or signs. This late presentation was not due to delay in referral or diagnosis. Furthermore the frequency of uncomplicated dyspepsia in our population aged less than 55 is less than the background population. Even a fivefold increase in the frequency of uncomplicated dyspepsia in gastric cancer in our sample would not approach the rate of uncomplicated dyspepsia in the community (greater than $20 \%$ ). Thus dyspepsia alone is not an indicator of malignancy and should not be a reason to screen for cancer in this age group.

It could be argued that as patients with dyspepsia are presenting with a symptom, performing endoscopy is not, strictly speaking, screening. However, if uncomplicated dyspepsia is no more likely to predict gastric cancer than no symptoms, there is no justification to look for gastric cancer in someone with uncomplicated dyspepsia. Thus, looking for gastric cancer in someone with uncomplicated dyspepsia is, strictly speaking, screening. There are, of course, other reasons for performing endoscopy and recently it has been shown in a randomised study that early endoscopy reduces prescribing, further consultation, and days lost from work in patients with dyspepsia. ${ }^{20}$ However, it is possible that if doctors and patients appreciated that gastric cancer without alarming features is extremely rare below the age of 55 , the reassurance of an endoscopy would not be so necessary in younger people.

In conclusion, we have shown in this study that gastric cancer below the age of 55, in a sample untainted by sampling bias, usually presents with recognisable alarm symptoms and/or signs. It should now be possible to reassure patients aged less than 55 with uncomplicated dyspepsia without the need for endoscopy and without significantly affecting the detection and staging of gastric cancer. This does not mean that endoscopy will no longer be required in this age group, just that it will be less important for the purpose of reassuring patients that they do not have cancer. Our findings will enable endoscopic resource to be liberated for investigation of older patients who have a much higher risk of cancer. Further studies are required to determine whether uncomplicated dyspepsia is a presenting symptom of gastric cancer in older people.

This paper was presented in abstract form to the British Society of Gastroenterology in September 1994. We would like to thank Mr MWL Gear, Dr J Barnes, and Dr SP Wilkinson whose Mr MWL Gear, Dr J Barnes, and Dr SP Wilkinson whose enabled us to do this study. We would also like to thank Dr R Walt for valuable advice in preparing the manuscript.

1 Office of Population Censuses and Surveys. Mortality statistics by cause. London: HMSO, 1991.

2 Hisamichi S. Screening for gastric cancer. World f Surg 1989; 13: 31-7.

3 Hallisey MT, Allum WH, Jewkes AJ, Ellis DJ, Fielding JWL. Early detection of gastric cancer. BMf 1990; 301: 513-5.

4 Sue-Ling HM, Johnston D, Martin IG, Dixon MF, Lansdown MRJ, McMahon MJ, et al. Gastric cancer: a Lansdown MRJ, McMahon MJ, et al. Gastric canc

5 Jones R, Lydeard S. Prevalence of symptoms of dyspepsia in the community. BMF 1989; 298: 30-2

6 Brown C, Rees WDW. Dyspepsia in general practice. $B M \mathcal{F}$ 1990; 300: 829-30.

7 Anonymous. Rationing in action. London: BMJ Publishing Group, 1993.

8 Gear MWL, Ormiston MC, Barnes RJ, Rocyn-Jones J, Voss GC. Endoscopic studies of dyspepsia in the community: an “open-access"service. BMF 1980; 280: 1135.

9 Gear MWL, Wilkinson SP. Open-access upper alimentary endoscopy. Br f Hosp Med 1989; 41: 438-44.

10 Allum WH, Powell DJ, McConkey CC, Fielding JWL. Gastric cancer: a 25-year review. Br J Surg 1989; 76: 535-40.

11 Watanabe H, Jass JR, Sobin LH. Histological typing of oesophageal and gastric tumours. Berlin: Springer Verlag, 1990.

12 Hermanek P, Sobin LH. TNM classification of malignant tumours. 4th edn, 2nd revision. Berlin: Springer Verlag, tumours.

13 Lauren P. The two histological main types of gastric carcinoma: diffuse and so called intestinal-type carcinoma. Acta Pathol Microbiol Scand 1965; 64: 31-49.

14 Parsonnet J, Friedman GD, Vandersteen DP, Chang Y, Vogelman JH, Orentreich N, Sibley RK. Helicobacter pylori infection and the risk of gastric carcinoma. $N$ Engl 7 Med 1991; 325: 1127-31.

15 Nomura A, Stemmerman GN, Chyou PH, Kato I, Perez-Perez GI, Blaser MJ. Helicobacter pylori infection and gastric carcinoma among Hawaii. N Engl f Med 1991; 325: 1132-6.

16 Correa P, Ruiz B. Helicobacter pylori and gastric cancer. In: Rathbone BJ, Heatley RW, eds. Helicobacter pylori and gastroduodenal disease, 2nd edn. Oxford: Blackwell Scientific gastroduodenal disease, 2nd edn.

17 Williams B, Luckas M, Ellingham JHM, Dain A, Wicks ACB. Do young patients with dyspepsia need investigation? Aancet 1988; ii: 1349-51.

18 Gillen D, El-Omar E, McColl KEL. Uncomplicated dyspepsia is a very rare presentation of GI malignancy under age 55 [abstract]. Gut 1996; 38: A33.

19 Canadian Task Force on the Periodic Health Examination. The periodic health examination. Can Med Assoc f 1979; 121: 1193-254.

20 Bytzer P, Hansen JM, Schaffalitsky de Muckadell OB. Empirical H2-blocker therapy or prompt endoscopy in management of dyspepsia. Lancet 1994; 343: 811-6. 